\title{
Band mapping in higher-energy x-ray photoemission: Phonon effects and comparison to one-step theory
}

\author{
L. Plucinski ${ }^{1,2}$ J. Minár, ${ }^{3}$ B. C. Sell,,${ }^{1,45}$ J. Braun, ${ }^{3,6}$ H. Ebert, ${ }^{3}$ C. M. Schneider, ${ }^{2}$ and C. S. Fadley ${ }^{1,2,4,7}$ \\ ${ }^{1}$ Materials Sciences Division, Lawrence Berkeley National Laboratory, Berkeley, California 04720, USA \\ ${ }^{2}$ Institute for Solid State Research, Research Center Jülich, D-52425 Jülich, Germany \\ ${ }^{3}$ Department of Chemistry and Biochemistry, Physical Chemistry Institute, Ludwig Maximillian University, Munich, D-81377 Munich, \\ Germany \\ ${ }^{4}$ Department of Physics, University of California Davis, Davis, California 95616, USA \\ ${ }^{5}$ Physics Department, Otterbein College, Westerville, Ohio 43081, USA \\ ${ }^{6}$ Hildesheim University, D-31141 Hildesheim, Germany \\ ${ }^{7}$ Department of Physics, University of Hamburg, D-22761 Hamburg, Germany
}

(Received 13 November 2007; revised manuscript received 20 April 2008; published 9 July 2008)

\begin{abstract}
We have studied the temperature dependence of $\mathrm{W}(110)$ soft $\mathrm{x}$-ray angle-resolved photoemission spectra excited at photon energies of 260 and $870 \mathrm{eV}$ and between 300 and $780 \mathrm{~K}$. The experimental results have been compared to both a free-electron final-state model and theoretical one-step model calculations of the photocurrent. At $300 \mathrm{~K}$, clear band dispersions can be observed in the data. The temperature dependence of the data can be analyzed qualitatively in terms of a direct-transition band-dispersion regime ("UPS" limit) versus a nondirect-transition density-of-states regime ("XPS" limit). The ratio between direct and nondirect transitions is estimated from a Debye-Waller factor, which for example at $h \nu=870 \mathrm{eV}$ predicts $70 \%$ direct transitions at $300 \mathrm{~K}$, and $41 \%$ at $780 \mathrm{~K}$, and these values qualitatively describe our data. Beyond this, the state-of-the-art one-step theoretical calculations reproduce well the band dispersions and matrix element effects in the measured spectra at room temperature. However, simulating the temperature dependence is more complicated, and including phonon effects via complex phase shifts accounts for the suppression of existing direct-transition features, but does not reproduce new, density-of-states-related background intensity which shows up in highertemperature experimental spectra. Finally, we also discuss the implications of this work for future experiments on other materials and at even higher photon energies up to $10 \mathrm{keV}$.
\end{abstract}

DOI: $10.1103 /$ PhysRevB.78.035108

PACS number(s): 71.20.-b, 79.60.-i

\section{INTRODUCTION}

There is a growing interest in extending valence electronic studies with angle-resolved photoemission (often referred to as "band mapping," but more correctly viewed as quasiparticle spectra) into the soft $\mathrm{x}$-ray ${ }^{1-3}$ and even hard $\mathrm{x}$-ray ${ }^{4}$ regimes. Such measurements take advantage of the greater photoelectron information depths at higher kinetic energies, thus probing more accurately bulk, rather than surface, electronic structure. Furthermore, it has been pointed out that three-dimensional, rather than two-dimensional, band structure and Fermi surfaces may be studied by going to higher photon energies. ${ }^{1}$ In comparison to low-energyband mapping, however, additional effects must be taken into account in both carrying out such measurements and in interpreting data. These include the increased angular resolution required to probe a small enough region in the Brillouin zone (BZ), the need to allow for the photon momentum in wave-vector conservation, and thermal effects due to phonon creation and annihilation during photoexcitation that smear out the specification of the initial wave vector. Papers discussing these issues in the $\sim 1.2-1.5 \mathrm{keV}$ range have been published some time ago, ${ }^{5,6}$ and a discussion of what is expected, at least qualitatively, upon going as high as the 5-10 keV regime has appeared more recently. ${ }^{7}$ It is useful in this discussion to think in terms of two limiting regimes: very low energies and/or temperatures in which band mapping is possible via direct or $k$-conserving transition (DTs) - the so-called UPS limit, and high energies and/or temperatures in which emission is fully averaged over the BZ to yield density-of-states (DOS) sensitivity-the XPS limit.

We will here focus on phonon effects as the most fundamental sample-associated limit to band mapping as energy or temperature is raised. As a test case, we use photoemission from tungsten, which has a broad valence-band distribution in energy, a high Debye temperature and mass that retains DT effects to higher temperatures, and which has in fact been used in prior studies of these effects. ${ }^{5}$ The present study goes beyond prior work in measuring detailed two-dimensional plots of binding energy vs wave vector as a function of temperature, with prior work on tungsten only considering selected single directions of emission and lower energy and angular resolution., ${ }^{5,6}$ Furthermore, we compare the experimental data with state-of-the-art one-step photoemission theory $^{8}$ which includes a precise evaluation of matrix element effects and an attempt to include phonon effects, while prior work used only simple direct-transition theory together with free-electron final states and a qualitative/ semiquantitative estimate of the fraction of DTs via suitable Debye-Waller (DW) factors ${ }^{5,6}$ with these being calculated from $W(T)=\exp \left[-1 / 3 g_{h k \ell}^{2}\left\langle U^{2}(T)\right\rangle\right]$, where $g_{h k l}$ is the magnitude of the bulk reciprocal-lattice vector involved in the direct transitions at a given photon energy and $\left\langle U^{2}(T)\right\rangle$ is the three-dimensional mean-squared vibrational displacement. Previous discussions of the theory of such phonon effects in valence photoemission have also appeared,,$^{5,6,9,10}$ but our 
one-step treatment is different in incorporating phonon effects via complex phase shifts to describe the atomic motion at each site, a procedure derived from low-energy electrondiffraction (LEED) theory and shown in various studies to provide a good description of the temperature dependence of LEED beam intensities. ${ }^{11}$ As additional background on such phonon effects, we note that prior theoretical analyses have indicated that, even in the assumption of only single phonon creation/annihilation during the photoemission process, the fractions of DT and nondirect-transition (NDT) intensities are expected to be more complex than can be estimated from $W(T)$ and $[1-W(T)]$, with one such analysis yielding the following form for the intensity at a given temperature $T$ and final-state wave vector $\vec{k}_{f}$ and energy $E:^{6}$

$$
I\left(\vec{k}_{f}, E_{f}, T\right)=W(T) I_{\mathrm{DT}}^{(0)}\left(\vec{k}_{f}, E_{f}, T\right)+I_{\mathrm{NDT}}\left(\vec{k}_{f}, E_{f}, T\right),
$$

where $I_{\mathrm{DT}}^{(0)}$ is the intensity for a rigid lattice and $I_{\mathrm{NDT}}$ is the intensity involving photon creation or annihilation, with specific form:

$$
\begin{aligned}
I_{\mathrm{NDT}}\left(\vec{k}_{f}, E, T\right) \propto & \frac{k_{f}}{v^{2}} \sum_{i} \delta\left(E_{f}-E_{i}-h \nu\right)\left|m_{\mathrm{if}}^{(0)}\right|^{2} \\
& \times \sum_{\vec{q}, \hat{\varepsilon}} \delta\left(\vec{k}_{f}-\vec{k}_{i}-\vec{g}_{h k \ell}-\vec{q}\right)\left\langle U_{\vec{q}, \hat{\varepsilon}}^{2}\right\rangle \\
& \times\left[\hat{\varepsilon} \bullet\left(\vec{k}_{f}-\vec{k}\right)_{i}\right],
\end{aligned}
$$

where $\nu$ is the photon frequency, $\left|m_{\mathrm{if}}^{(0)}\right|^{2}$ is the one-electron matrix element connecting the initial state at $E_{i}, \vec{k}_{i}$ with the final state at $E_{f}, \vec{k}_{f}, \vec{q}$ is the phonon wave vector, $\hat{\varepsilon}$ is the polarization of the phonon vibration, $\left\langle U_{\vec{q}, \hat{\varepsilon}}^{2}\right\rangle$ is the meansquared amplitude of the phonon mode, and the $\delta$ functions allow for energy and wave-vector conservation. The energy $E$ is here measured inside the surface, and thus is higher than the measured energy by the inner potential $V_{0}$. From these equations, it is clear that the DT intensity should decrease as the DW factor, but the increase in the NDT intensity can be more complex, particularly if multiphonon effects beyond these equations are included. ${ }^{6,9,10}$ However, since the vibrational amplitudes will have maximum values for $\vec{q}$ values near zero, it is expected that phonon effects will selectively increase intensity near the allowed DT transitions, ${ }^{6}$ and this could lead to an effective decrease in the observed DT component with increasing temperature that is less severe than that expected from $W(T)$.

\section{EXPERIMENT}

Our measurements were carried out on a (110)-oriented tungsten crystal at beamline 4.0.2 of the Advanced Light Source in Berkeley, using the Multi-Technique Spectrometer/ Diffractometer located there. ${ }^{12}$ A slightly simplified view of the experimental geometry is shown in Fig. 1. The exciting synchrotron radiation was $p$ polarized and chosen to be at $h \nu=260 \mathrm{eV}$ and $870 \mathrm{eV}$, the angle between photon incidence and electron exit was fixed at $70^{\circ}$. These two energies were chosen such that, as estimated via a free-electron final-state model for direct transitions, we would be sampling a curve

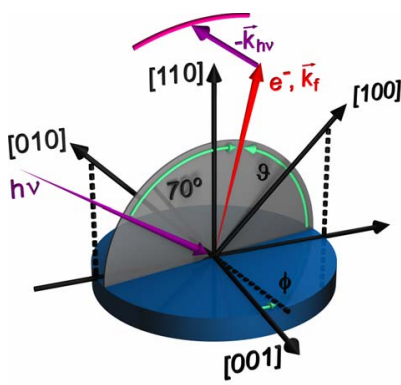

$$
\begin{gathered}
\overrightarrow{\boldsymbol{k}}_{f}=\overrightarrow{\boldsymbol{k}}_{i}+\overrightarrow{\boldsymbol{g}}_{h \boldsymbol{k} \ell}+\overrightarrow{\boldsymbol{k}}_{h v}+\overrightarrow{\boldsymbol{g}}_{\text {surf }}+\vec{q}_{\text {phonon }} \\
\overrightarrow{\boldsymbol{k}}_{\boldsymbol{i}} \approx \overrightarrow{\boldsymbol{k}}_{f}-\overrightarrow{\boldsymbol{k}}_{h v}-\overrightarrow{\boldsymbol{g}}_{h \boldsymbol{k} \ell}
\end{gathered}
$$

FIG. 1. (Color online) The experimental geometry, with various key elements, and two key wave-vector conservation equations, indicated. The approximate curve sampled in our angle-resolved data is also shown. The angles $\theta$ and $\phi$ here refer to those inside the surface, after allowance for slight refraction effects on $\theta$ due to the inner potential.

of $k$ points passing close to the $\Gamma$ point. The sample orientation was adjusted after allowing for photon momentum so as to as nearly as possible sample points along the $\Gamma$-to- $N$ line in the BZ. The emission angle was near normal, with $\theta$ near to, but less than $90^{\circ}$, and the azimuthal orientation was such that $\phi$ was near $0^{\circ}$ also, with the excitation-and-emission plane lying close to the plane containing the [110] and [001] directions. Small effects of electron refraction at the surface due to the inner potential $V_{0}$, which we take to be $15 \mathrm{eV}$ for $\mathrm{W}(110)$, have been allowed for in our analysis, and they lead to slight differences of $\theta$ between electron propagation inside and outside the surface $\left(<0.5^{\circ}\right.$ for $260 \mathrm{eV}$ excitation and $<0.3^{\circ}$ for $870 \mathrm{eV}$ excitation). A Scienta electron spectrometer with SES2002 performance was used to accumulate angle-resolved spectra with a standard two-dimensional (2D) detection scheme. The total-energy resolution was $150 \mathrm{meV}$ at $h \nu=260 \mathrm{eV}$ and $400 \mathrm{meV}$ at $870 \mathrm{eV}$, and the average angular resolution was approximately $0.5^{\circ}$. The sample was cleaned in situ using standard high-temperature oxygen exposure and annealing, with cleanliness verified via $\mathrm{O} 1 \mathrm{~s}$ and W $4 f$ core-level photoemission ${ }^{13}$ using both synchrotron radiation and a nonmonochromatized $\operatorname{Mg} K \alpha$ source at $h \nu$ $=1253.6 \mathrm{eV}$ for excitation. Some angle-resolved photoemission (ARPES) spectra were also obtained using this standard XPS source. The sample orientation was determined to within $\sim \pm 0.5^{\circ}$ using a combination of LEED, $x$-ray photoelectron diffraction, and laser reflection from the surface. At the energies under consideration, this uncertainty corresponds to $5 \%$ of the distance from $\Gamma$-to- $N$ in the tungsten BZ for $h \nu=260 \mathrm{eV}, 9 \%$ of this distance for $870 \mathrm{eV}$, and $11 \%$ of this distance for the maximum energy of $1253.6 \mathrm{eV}$ mentioned above. The magnitude of the photon momentum correction corresponds to $9 \%$ of $\Gamma$-to- $N$ for $260 \mathrm{eV}, 31 \%$ for 870 $\mathrm{eV}$, and $45 \%$ for $1253.6 \mathrm{eV}$. We have allowed for both of these effects for all energies studied in comparing experiment to theory. Although our sample had a slight misalignment relative to the symmetry axis of the spectrometer entrance aperture of approximately $1.25^{\circ}$, with this misalignment determined to within the accuracy mentioned above, we were able by fine tuning $\theta$ and $\phi$ to align it such 
(a) $300 \mathrm{~K} \mathrm{~W}=0.90$

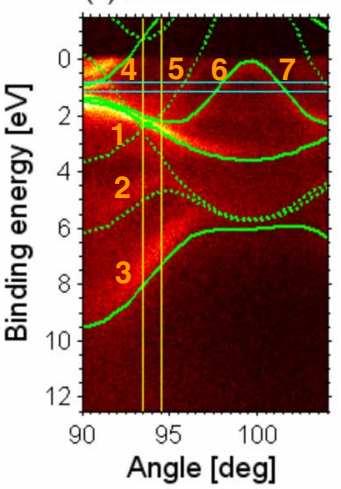

(e) EDC cut

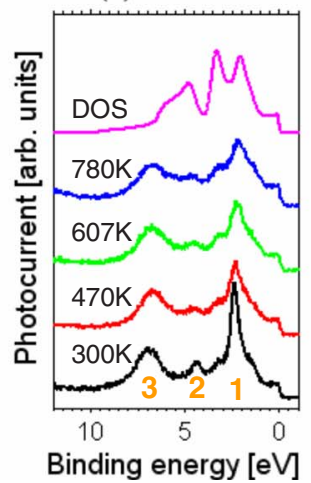

(b) $470 \mathrm{~K} \mathrm{~W}=0.85$

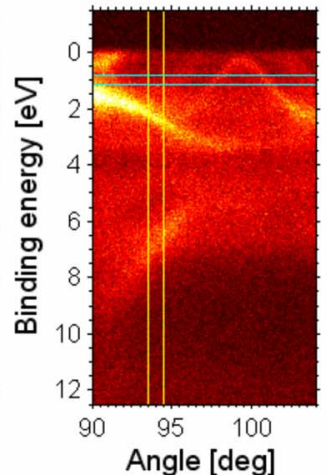

(f) MDC cut

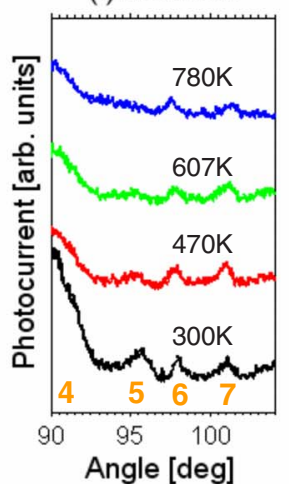

(c) $607 \mathrm{~K} \mathrm{~W}=0.81$

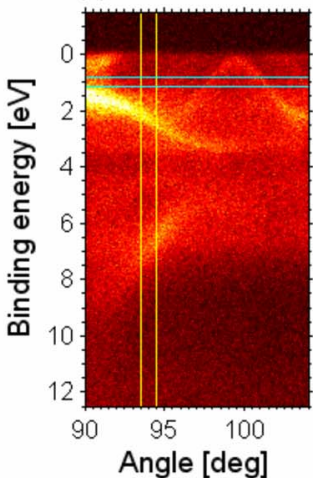

(d) $780 \mathrm{~K} \mathrm{~W}=0.77$

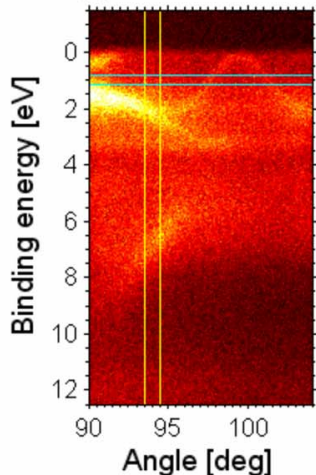

FIG. 2. (Color online) (a)-(d) Plots of intensity versus angle of emission for $260 \mathrm{eV}$ excitation from the valence bands of W(110) and for an emission angle that samples initial states roughly along the $\Gamma$-to- $N$ line in the Brillouin zone, and for four temperatures of $300,470,607$, and $870 \mathrm{~K}$. In (a) also the transitions allowed with free-electron final states are shown, solid lines are primary cone bands and the dashed lines surface umklapp bands with $\overrightarrow{\mathbf{g}}=\langle 112\rangle \pi / a$. The numbers label certain features that also appear in (e) and (f). (e) The temperature dependence of energy distribution curves as integrated over 20 channels in angle as indicated in as indicated in (a)-(d). A comparison to the $W$ density of states (DOS), as broadened by experimental resolution of $150 \mathrm{meV}$ is also shown in the topmost curve. (f) The temperature dependence of momentum distribution curves at one selected energy, again as indicated in (a)-(d). (g) The average locus of points in the BZ sampled in this data, assuming direct transitions and free-electron final states.

that the locus of points probed in the BZ lies close to selected high-symmetry points. In achieving the latter, the final orientations were slightly different for 260 and $870 \mathrm{eV}$ excitations, with the major factor in this being the different magnitude of the photon momentum correction.

\section{RESULTS AND DISCUSSION}

In Figs. 2(a)-2(d), we show ARPES results with $260 \mathrm{eV}$ excitation energy and acquired at four different sample temperatures: 300, 470, 607, and $780 \mathrm{~K}$. The relevant DW factors are also indicated, as calculated from the Debye model and a Debye temperature of tungsten of $400 \mathrm{~K}{ }^{5}$ We can clearly see the band structure of $W$ in all of these spectra, but with significant smearing of features and an increase of intensity in nonsimple band positions as the temperature is raised.

We have analyzed this data in first approximation by requiring wave-vector conservation according to ${ }^{5}$

$$
\vec{k}_{f}=\vec{k}_{i}+\vec{g}_{h k l}+\vec{k}_{h \nu}
$$

where the $\vec{k}$-conservation condition in Eq. (1) has been modified to allow for the non-negligible photon momentum at higher excitation energies, by assuming free-electron final states for which the energy inside the solid is $E_{f}\left(\vec{k}_{f}\right)$ $=\hbar^{2} k_{f}^{2} / 2 m_{e}=E_{\mathrm{kin}}-V_{o}$, where $m_{e}$ is the mass of the electron, $E_{\text {kin }}$ is the kinetic energy outside the surface, $V_{0}$ is the inner potential of $15 \mathrm{eV}$, and by using initial-state energies $E_{i}\left(\bar{k}_{i}\right)$ from a band structure calculated using the WIEN2K code. ${ }^{14}$ Note that this analysis is inherently done in three dimensions, such that the $x, y$, and $z$ (vertical) components of $\vec{k}_{f}$ are all conserved. Although there is uncertainty in the $z$ component due to the uncertainty in $V_{0}$, this is at maximum $\sim \pm 2 \mathrm{eV}$, which corresponds to only about $0.07 \AA^{-1}$ at this energy, or $1 / 28^{\text {th }}$ of the $\Gamma$-to- $X$ distance in the BZ. The solid and dotted curves superimposed on the experimental data at $300 \mathrm{~K}$ correspond to this simple analysis. The solid curves correspond to transitions associated in normal emission with $\vec{g}_{h k l}=[660] \pi / a$, and the dashed curves representing additional transitions that are possible due to surface umklapp effects via the set of $g$ vectors $[-112] \pi / a$. Figure $2(\mathrm{~g})$ indicates the average curve in the BZ that is responsible for these spectra, as derived from this free-electron model. Although this curve represents only a projection onto the (001) plane, three-dimensional views confirm that we are here looking roughly along the line $\Gamma$-to- $N$, with our experimental align- 
(a) $300 \mathrm{~K}$

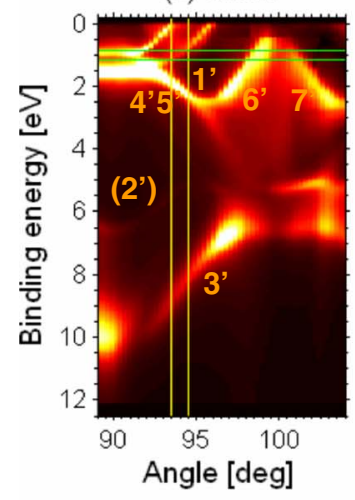

(b) $470 \mathrm{~K}$

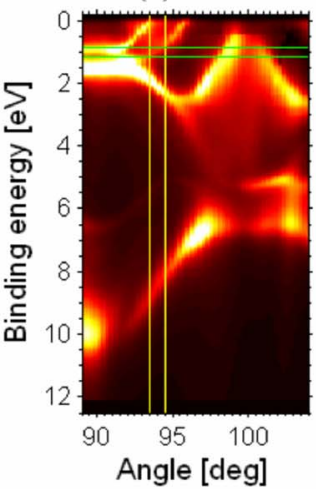

(e) EDC cut

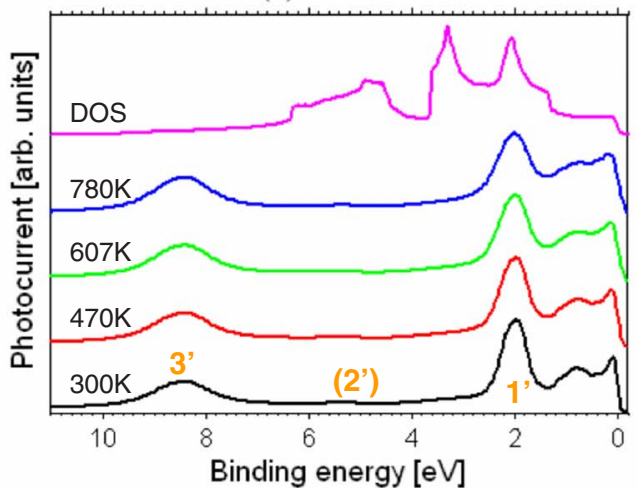

(c) $607 \mathrm{~K}$

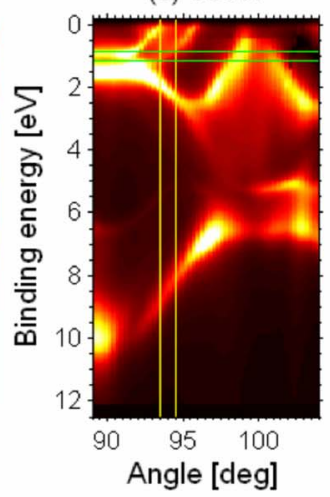

(d) $780 \mathrm{~K}$

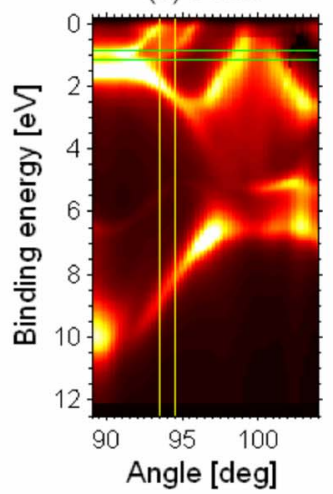

(f) MDC cut

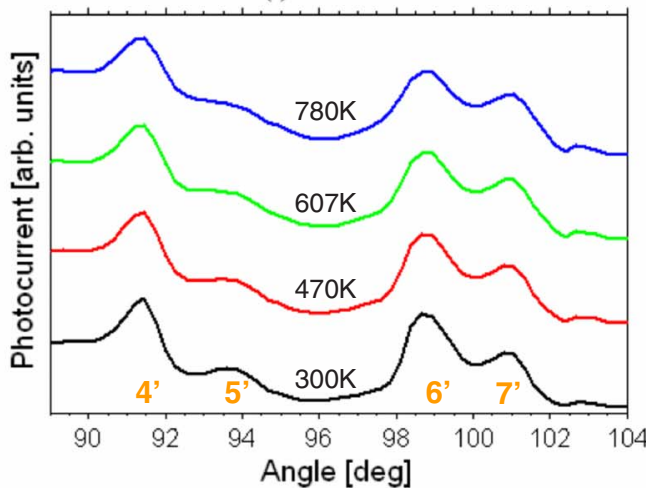

FIG. 3. (Color online) As in Fig. 2, but for one-step photoemission calculations including temperature effects via complex phase shifts. See text for details.

ment having been empirically adjusted to yield this. It is obvious that the agreement is very good, which proves that band mapping is indeed possible at $260 \mathrm{eV}$, an energy higher than used in most prior ARPES work. As a simple expedient in these calculations, the kinetic energy in the final state from which the final $k$ vector is derived has been assumed to be that for emission from the Fermi level, rather than using the trial and error scheme of prior work in which the kinetic energy is adjusted for each allowed band DT; ${ }^{4,5}$ this simplification is the reason for the slight disagreement between theory and experiment near the valence-band bottom.

Also shown in Fig. 2(e) are vertical cuts through the 2D data, as integrated over 20 angle channels, to illustrate more directly the change in energy distribution curves (EDCs) with temperature. For reference, the top curve in Fig. 2(e) is a theoretical density of states for $W$, broadened by our estimated experimental resolution; this would approximate the XPS limit of spectra. In Fig. 2(f), horizontal cuts through the data are shown to illustrate the change in momentum distribution curves (MDCs). Various features in both the 2D spectra, EDCs, and MDCs are labeled 1-7 for clarity. It is clear in both the 2D plots and the EDCs and MDCs that temperature smears out the spectral features. This trend would lead ultimately to constant MDCs and an EDC that reflects a matrix element-modulated density of states: the XPS limit. However, another important observation from Figs. 2(a)-2(f) is that not all bandlike features are equally affected by phonon broadening, with the more free-electron-looking bands leading to the peaks labeled 6 and 7 persisting as sharp even at the highest temperature of our measurements. This is consistent with the expectation that more delocalized electronic states will have a weaker interaction with phonons, as discussed in prior theoretical papers. ${ }^{15,16}$ It is thus clear that a correct treatment of the electron-phonon coupling involved can lead to differing degrees of sensitivity to increased temperature for different bands. This observation concerning weaker coupling of free-electron-like bands to phonons may also explain why a greater degree of temperature dependence was not seen in a recent soft x-ray ARPES study of aluminum. ${ }^{10}$

In discussing this data, it is also of interest to compare it to a classic theoretical and experimental ARPES study of tungsten in normal emission and at much lower photon energies of 7.7 to $21.2 \mathrm{eV} .{ }^{17}$ Due to the much lower energy, the results in this case are much more surface sensitive than ours, and they were recorded for a fixed emission direction and at several photon energies rather than at a couple of photon energies with sensitivity to a range of emission directions as in our data. These measurements thus at best sample a single point in the BZ for each photon energy, or at most a small excursion along $k_{z}$ due the change in energy within a given spectrum. By contrast, our BZ sampling at each photon energy represents much more detailed data in direction and thus $\vec{k}_{f}$. The influence of surface states near the Fermi level is also much more important in this prior data. It is thus difficult to quantitatively compare these results with our own, but we see no fundamental disagreements between these two very different types of ARPES measurements. 
(a) $300 \mathrm{~K} \mathrm{~W}=0.70$

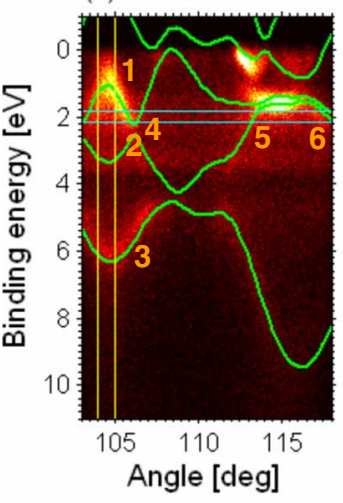

(e) EDC cut

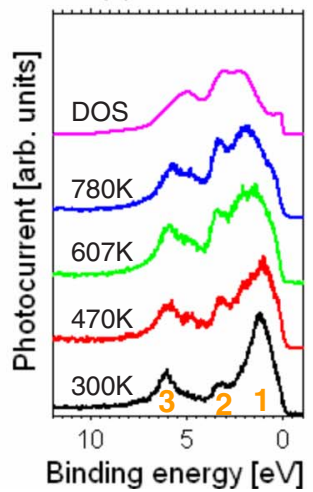

(b) $470 \mathrm{~K} \mathrm{~W}=0.58$

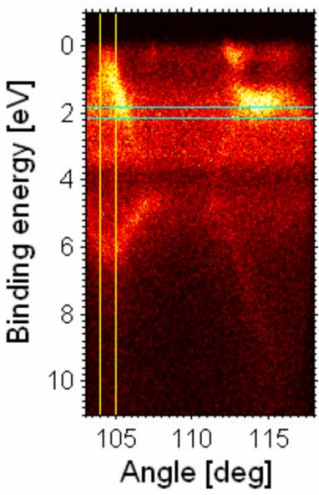

(f) MDC cut

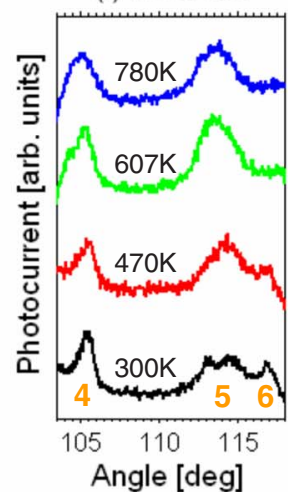

(c) $607 \mathrm{~K} \mathrm{~W}=0.50$

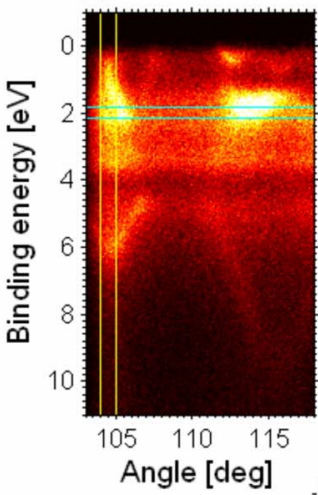

(d) $780 \mathrm{~K} \mathrm{~W}=0.41$
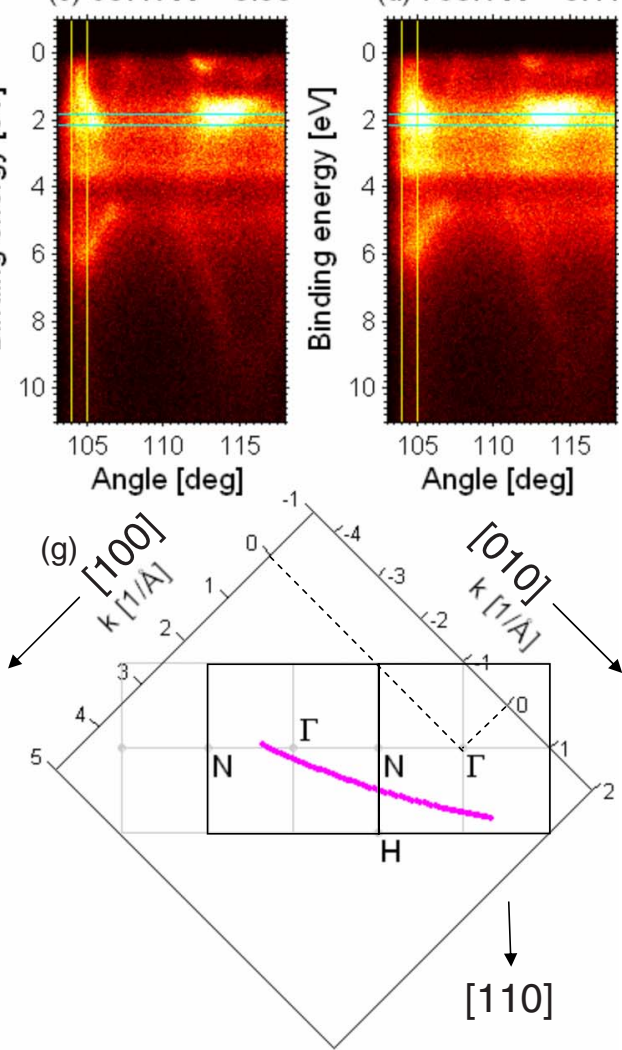

FIG. 4. (Color online) As in Fig. 2 but for $870 \mathrm{eV}$ excitation and an emission angle that approximately samples the $N$-to- $\Gamma$ line in the Brillouin zone. In (e) we show for comparison the $W$ density of states (DOS), as broadened by experimental resolution of $400 \mathrm{meV}$.

As an additional point concerning our data, it is possible from prior theoretical studies, ${ }^{17,18}$ as well as from our own calculations with the WIEN2K code, ${ }^{19}$ to assign approximate orbital characters to the different bands seen. For the data in Fig. 2, the points labeled $1,2,4$, and 5 are strongly $d$ in character, point 3 is mostly $s$ in character, and points 6 and 7 are a mix of $p$ and $d$ character.

Figures 3(a)-3(d) display analogous results from one-step photoemission theoretical calculations for the $260 \mathrm{eV}$ cases shown in Figs. 2(a)-2(d), with similar EDC and MDC cuts in Figs. 3(e) and 3(f). The full details concerning this theoretical approach appear elsewhere. ${ }^{8}$ Various features in the theoretical results have been labeled $1^{\prime}-7^{\prime}$ in such a way as to maximally correspond to the experimental results. We again note good agreement between theory and experiment for the $300 \mathrm{~K}$ results, including in particular a generally correct prediction of the relative intensities of various features. Although, a feature 2 in experiment appears to be stronger than $2^{\prime}$ in theory, this is primarily due to the different color scales of the two plots. However, feature 2 is due to surface umklapp effects, and it may be that these are underestimated in the theoretical calculations. The theoretical calculations, which are done in a layer KKR formalism, ${ }^{8}$ in fact implicitly allow for surface umklapp scattering process. Furthermore, theory does not predict the smearing of features at higher temperatures in either the 2D plots or the EDCs or the MDCs that is seen in experiment, but rather shows only a simple suppression of the direct-transition features.
We now turn to the measurements at $h \nu=870 \mathrm{eV}$, with Figs. 4 and 5 presenting experiment and theory in the same format as Figs. 2 and 3 for $h \nu=260 \mathrm{eV}$, respectively. For this higher energy, the same sort of uncertainty in $V_{0}$ corresponds to only about $0.04 \AA^{-1}$, or $1 / 50^{\text {th }}$ of the $\Gamma$ to $X$ distance in the BZ. Also for this higher-energy data, the smearing due to phonon effects is much more pronounced than at $260 \mathrm{eV}$, as expected from a Debye-Waller argument, with the relevant values of $W$ being given in each of panels (a)-(d).

The solid curves in Fig. 4(a) also demonstrate once again that there is excellent agreement between theory based on $\vec{k}$ conservation in a simple free-electron final-state model and experiment. For this case, the $\vec{g}_{h k l}$ involved for normal emission is [ $\left[\begin{array}{lll}10 & 10 & 0\end{array}\right] \pi / a$, but in fact our sample was rotated slightly away from the [110] surface normal and toward the [100] direction by about $11^{\circ}$ so that the $\left[\begin{array}{lll}12 & 8 & 0\end{array}\right] \pi / a$ vector was the primary one supporting direct transitions. As a general comment, however, the angular width of approximately $15^{\circ}$ spanned by the detector implies that more than one $\vec{g}_{h k l}$ will often be involved at this higher energy. That is, $\left|\vec{k}_{f}\right|$ $\cong 15.4 \AA^{-1}$ and this vector will thus sweep over $\sim(15.4)$ $\times 2 \times \sin \left(7.5^{\circ}\right) \cong 4.0 \AA^{-1}$ in a single detector image. This sweep of $4.0 \AA^{-1}$ is about the diameter of the BZ, with e.g., the distance from $\Gamma$ to $X$ being $1.99 \AA^{-1}$. Overall, this threedimensional DT model, whose average locus of $k$-point sampling is indicated in Fig. 4(g) (again projected onto the (001) plane), accounts very well for most of the features seen in the spectra. 
(a) $300 \mathrm{~K}$

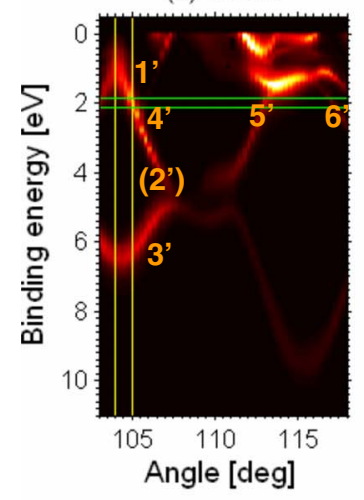

(b) $470 \mathrm{~K}$

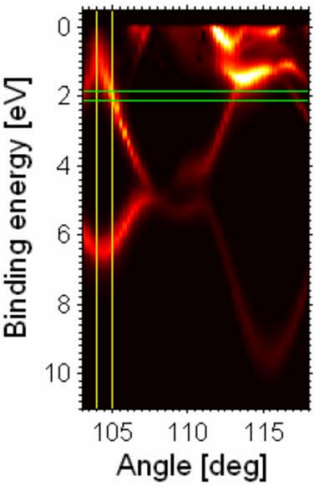

(e) EDC cut

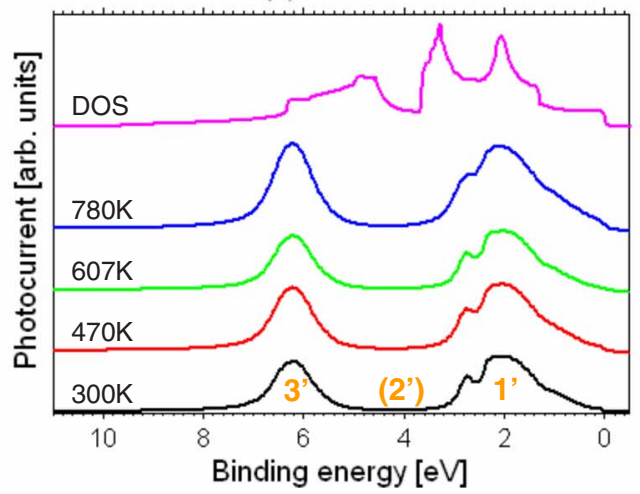

(c) $607 \mathrm{~K}$

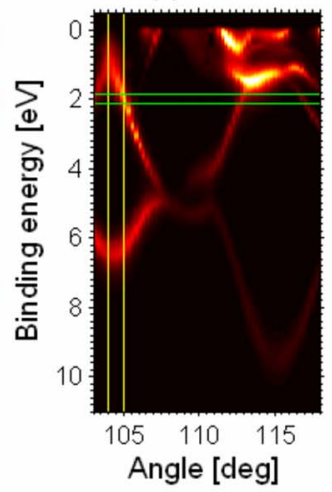

(d) $780 \mathrm{~K}$

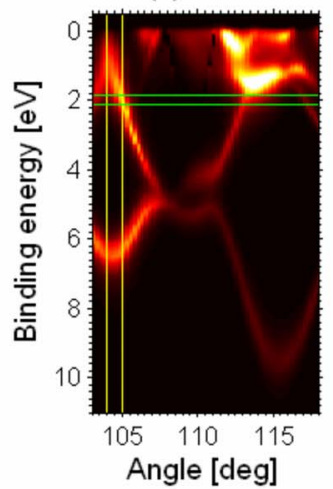

(f) MDC cut

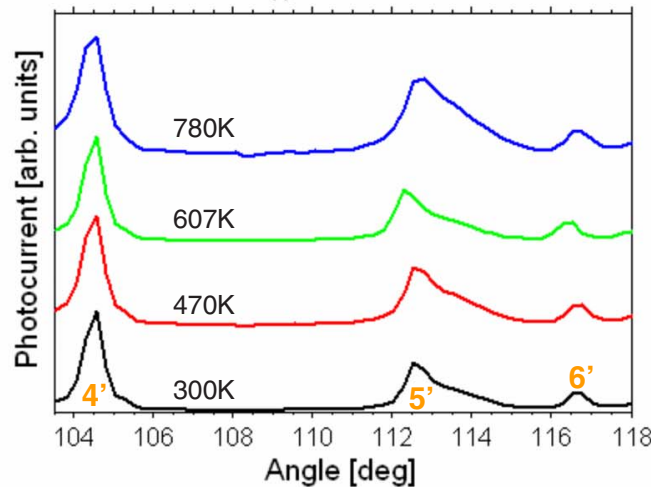

FIG. 5. (Color online) As in Fig. 3 but for a choice of $890 \mathrm{eV}$ excitation that was found to yield optimum agreement with experiment.

Just as for the data in Fig. 2, it is possible to assign approximate orbital characters to the different bands seen. For the data in Fig. 4, the points labeled 1, 2, 4, 5, and 6 are strongly $d$ in character, with point 3 being more of a mixture of $d$ and $s$ character. The free-electron-like band extending down to about $9 \mathrm{eV}$ is strongly $s$ in character.

Now, considering the comparison of experiment with onestep theory in Fig. 5, we have first found that in order to obtain optimum agreement between the two, it is necessary to increase the photon energy from 870 to $890 \mathrm{eV}$ in the theoretical calculations. That is, the theoretical spectra at $h \nu=890 \mathrm{eV}$ compare best with the experimental ones at 870 $\mathrm{eV}$. This shift has its origin in an underestimation of the energy of the unoccupied bands due to the local spin-density approximation. ${ }^{20}$ Another way of correcting this deviation would be to use the so-called "scissor operator" to rigidly shift the unoccupied bands such that the best agreement is obtained empirically; but this is essentially equivalent to what we have done. Figure 5 also indicates that there is again good agreement between theory and experiment for $300 \mathrm{~K}$ as to the features seen, and to their relative intensities. Thus, for both $h \nu=260 \mathrm{eV}$ and $870 \mathrm{eV}$, the matrix element effects as calculated in theory correctly represent our experimental data. One point of discrepancy, however, is that the weak feature labeled 2 in Fig. 2 is not seen as strongly in theory; again, this could be a matter of slightly different alignments between the two, and such discrepancies need further study.

Even though the experimental data in Fig. 4 show more pronounced smearing of features in both EDCs and MDCs as the temperature is raised, some remnant direct-transition be- havior is clearly still present at $780 \mathrm{~K}$; the EDC at the highest temperature has not converged to a DOS-like curve such as that shown in Fig. 4(e), and the corresponding MDC still has structure in it. (In the XPS limit all MDCs would be flat lines.) This might be expected from the DW factor of 0.41 calculated for this energy and temperature (i.e., an estimate of $\sim 41 \%$ of transitions being direct).

Comparing Figs. 4 and 5 with respect to temperature dependence again indicates that the inclusion of phonon effects via complex phase shifts as done in these calculations does not correctly account for the smearing in $k$ space that is seen in experiment. In both Figs. 3 and 5, theory does correctly predict a diminution of the features expected due to direct transitions, but there is no significant broadening of features in the EDCs or MDCs in theory and no prediction of the relatively smooth background introduced by phonon effects. Note also that this phonon-induced background in the experimental data of Fig. 4 still shows a lower-intensity horizontal band at about $4 \mathrm{eV}$ binding energy which is associated with a "pseudo band gap" in the tungsten density of states [e.g., see Fig. 4(e)]. This limitation of the complex-phase-shift method of treating vibrational displacements is due to the fact that it does not correctly allow for the creation/ annihilation of phonons, with their associated momenta then influencing wave-vector conservation. Rather, this method acts primarily to suppress the relative intensity of existing DT features, perhaps introducing a slight broadening of them in angle or energy at higher temperatures, as can be seen from a comparison of the EDCs and MDCs in Figs. 3 and 5. More sophisticated approaches based on allowing for such 


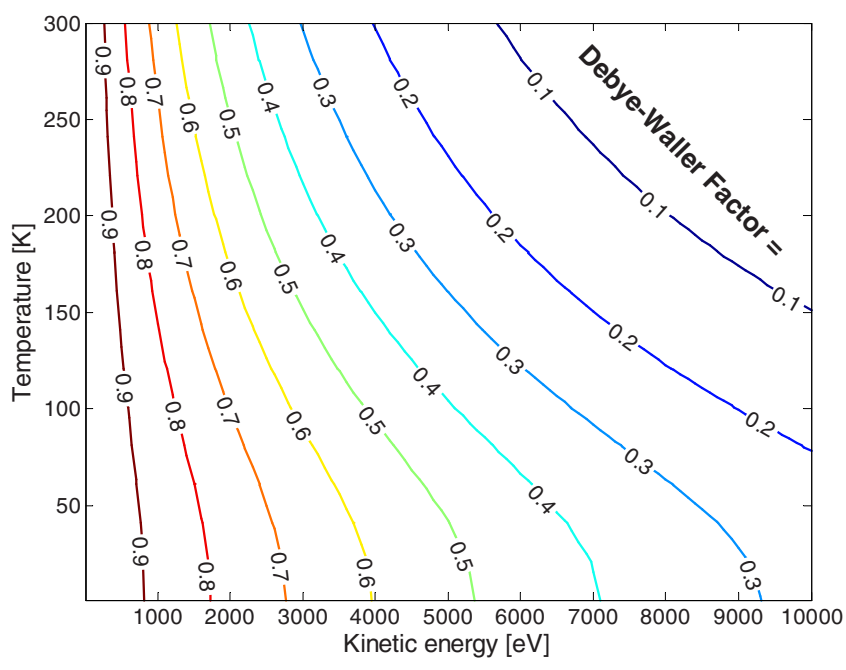

FIG. 6. (Color online) Debye-Waller factors for valence-band photoemission from $W$ at various temperatures over $0-300 \mathrm{~K}$ and electron kinetic energies over $0-10 \mathrm{keV}$. These permit a rough estimate of the fraction of transitions yielding simple band mapping features.

effects, as well as changes in both the electronic energy levels and the matrix elements due to atomic vibrational displacements, have been discussed ${ }^{6,9,10}$ and applied with several simplifying assumptions to the simple free-electron metal aluminum, ${ }^{9,10}$ but these approaches have to date not been implemented in a way that can be applied quantitatively to a system such as tungsten.

We also note as another aspect of considering band mapping at higher energies that the 2D imaging properties of our spectrometer lens have permitted measuring band profiles for $W$ at room temperature and $\mathrm{LN}$ temperature, even with a standard, nonmonochromatized, source at 1,253.6 eV, ${ }^{21}$ although we do not show this data here.

Finally, we consider what might be expected if the photon energy is taken to much higher values up to $10 \mathrm{keV}$, again for the example case of tungsten. At this point, we can only make use of the DW factors to estimate the fraction of transitions that are direct, and a family of curves for different temperatures over $0-300 \mathrm{~K}$ and electron kinetic energies over $0-10 \mathrm{keV}$ are shown in Fig. 6. From these curves, it is clear that, if we use the rough criterion of at least $50 \%$ direct transitions (as e.g., is approximately the case for Fig. 4(c), i.e., $870 \mathrm{eV}-607 \mathrm{~K}$ ), then at least for $W$, one can work at 300 $\mathrm{K}$ with photon energies up to about $1.7 \mathrm{keV}$, and with cooling to $4 \mathrm{~K}$, with photon energies up to about $5.4 \mathrm{keV}$. Zero-point motion leads to all of the curves in this figure being nearly vertical below about $20 \mathrm{~K}$, so that ultralow temperatures may not in fact be needed to take maximum advantage of higherenergy band mapping. Of course, as we have already noted, $W$ is a particularly robust choice for ARPES at high energies, and curves similar to those in Fig. 6 would have to be cal- culated or estimated for any other material. But we believe these data overall suggest the possibility of doing useful band mapping even for other less robust materials with photon energies up to the $1-3 \mathrm{keV}$ regime, provided cryogenic cooling is employed.

\section{CONCLUSIONS}

In summary, we have shown that meaningful band mapping can be done for the test case of $W$ at room temperature with photon energies up to $870 \mathrm{eV}$ (or in fact up to at least $1,253 \mathrm{eV}$ in data not shown here ${ }^{21}$ ), with features in experiment that are semiquantitatively predicted by assuming freeelectron final states. Comparing room-temperature experiment with one-step model photoemission theory for both 260 and $870 \mathrm{eV}$ is found to yield very good agreement as to almost all spectral features and relative intensities. The temperature dependence of the experimental data on going up to $780 \mathrm{~K}$ shows smearing in both EDCs and MDCs due to phonon effects, but with delocalized electronic states exhibiting much less sensitivity. However, theory in which phonon effects are incorporated only via complex phase shifts is not found to reproduce the smearing of the spectral features, and this indicates that a more accurate approach in which phonon creation and annihilation are taken into account in the photoemission process is needed to adequately describe such data. ${ }^{6,9,10}$ Finally, we have provided via Debye-Waller factors a rough estimate of the regime in temperature and energy over which band mapping should be possible for $W$, and these results suggest that useful ARPES measurements should be possible with cryogenic cooling and for photon energies up to at least a few $\mathrm{keV}$ range for other materials, However, further experimental and theoretical studies on this point are clearly called for, and a similar temperaturedependent soft x-ray study of ARPES from Ag has in fact been carried out recently; ${ }^{22} \mathrm{Ag}$ is a system for which phonon effects are expected to be much more important, due to its lower Debye temperature.

\section{ACKNOWLEDGMENTS}

The experimental work described here was supported by the Director, Office of Science, Office of Basic Energy Sciences, Materials Science and Engineering Division, U. S. Department of Energy under Contract No. DE-AC0376 SF00098 (L. P. and C. S. F.), and the one-step photoemission theoretical calculations by the German BMBF (Bundesministerium für Bildung und Forschung) under Contract No. FKZ-05-KS1WMB/1 (J. M., J. B., and H. E.). We are also grateful to E. Rotenberg for helpful comments at the initiation of this study. One of us (C. S. F.) also gratefully acknowledges the support of the Alexander von Humboldt Foundation and the Helmholtz Association, and the Berliner Elektronenspeicherring-Gesellschaft (BESSY). 
${ }^{1}$ B. Nielsen, Z. Li, S. Lizzit, A. Goldoni, and Ph. Hoffmann, J. Phys.: Condens. Matter 15, 6919 (2003).

${ }^{2}$ M. Yano, A. Sekiyama, H. Fujiwara, T. Saita, S. Imada, T. Muro, Y. Onuki, and S. Suga, Phys. Rev. Lett. 98, 036405 (2007), and references therein.

${ }^{3}$ N. Kamakura, Y. Takata, T. Tokushima, Y. Harada, A. Chainani, K. Kobayashi, and S. Shin, Phys. Rev. B 74, 045127 (2006).

${ }^{4}$ A. Yamasaki, A. Sekiyama, S. Imada, M. Tsunekawa, A. Higashiya, A. Shigemoto, and S. Suga, Nucl. Instrum. Methods Phys. Res. A 547, 136 (2005).

${ }^{5}$ Z. Hussain, C. S. Fadley, S. Kono, and L. F. Wagner, Phys. Rev. B 22, 3750 (1980).

${ }^{6}$ R. C. White, C. S. Fadley, M. Sagurton, and Z. Hussain, Phys. Rev. B 34, 5226 (1986).

${ }^{7}$ C. S. Fadley, Nucl. Instrum. Methods Phys. Res. A 547, 24 (2005), and references therein.

${ }^{8}$ J. Braun, Rep. Prog. Phys. 59, 1267 (1996).

${ }^{9}$ M. A. Vicente Alvarez, H. Ascolani, and G. Zampieri, Phys. Rev. B 54, 14703 (1996); C. Søndergaard, P. Hofmann, C. Schultz, S. M. Moreno, J. E. Gayone, M. A. Vicente Alvarez, G. Zampieri, S. Lizzit, and A. Baraldi, ibid. 63, 233102 (2001).

${ }^{10}$ P. Hofmann, C. Sondergaard, S. Agergaard, S. V. Hoffmann, J. E. Gayone, G. Zampieri, S. Lizzit, and A. Baraldi, Phys. Rev. B 66, 245422 (2002).

${ }^{11}$ C. G. Larsson and J. B. Pendry, J. Phys. C 14, 3089 (1981).
${ }^{12}$ C. S. Fadley, M. A. Van Hove, Z. Hussain, and A. P. Kaduwela, J. Electron Spectrosc. Relat. Phenom. 75, 273 (1995).

${ }^{13}$ T. M. Duc, C. Guillot, Y. Lassailly, J. Lecante, Y. Jugnet, and J. C. Vedrine, Phys. Rev. Lett. 43, 789 (1979).

${ }^{14}$ P. Blaha, K. Schwarz, G. K. H. Madsen, D. Kvasnicka, and J. Luitz, An Augmented Plane Wave Plus Local Orbitals Program for Calculating Crystal Properties (Karlheinz Schwarz, Technical Universität Wien, Austria, 2000).

${ }^{15}$ G. D. Gaspari and B. L. Gyorrfy, Phys. Rev. Lett. 28, 801 (1972).

${ }^{16}$ W. E. Pickett, Phys. Rev. B 25, 745 (1982).

${ }^{17}$ N. E. Christensen and B. Feuerbacher, Phys. Rev. B 10, 2349 (1974); B. Feuerbacher and N. E. Christensen, ibid. 10, 2373 (1974).

${ }^{18}$ V. P. Zhukov and V. A. Gubanov, Solid State Commun. 56, 51 (1985).

${ }^{19}$ L. Plucinski (unpublished).

${ }^{20} \mathrm{~J}$. Braun, in Band-Ferromagnetism: Ground-State and FiniteTemperature Phenomena, Lecture Notes in Physics Vol. 580, edited by K. Baberschke, M. Donath, and W. Nolting (SpringerVerlag, Berlin, 2001), pp. 341-355.

${ }^{21}$ L. Plucinski, C. Papp, I. Preda, M. Malvestuto, M. W. West, M. Press, C. M. Schneider, and C. S. Fadley (unpublished).

${ }^{22}$ F. Venturini, J. Minar, J. Braun, H. Ebert, and N. B. Brookes, Phys. Rev. B 77, 045126 (2008). 